University of Wollongong

Research Online

Faculty of Engineering and Information

Faculty of Engineering and Information

Sciences - Papers: Part A

Sciences

$1-1-2013$

\title{
A compressed sensing method for complex-valued signals with application to through-the-wall radar imaging
}

Fok Hing Chi Tivive

University of Wollongong, tivive@uow.edu.au

Abdesselam Bouzerdoum

University of Wollongong, bouzer@uow.edu.au

Follow this and additional works at: https://ro.uow.edu.au/eispapers

Part of the Engineering Commons, and the Science and Technology Studies Commons

Research Online is the open access institutional repository for the University of Wollongong. For further information contact the UOW Library: research-pubs@uow.edu.au 


\title{
A compressed sensing method for complex-valued signals with application to through-the-wall radar imaging
}

\author{
Abstract \\ In this paper, we present a compressed sensing method for complex-valued signals based on multiple \\ measurement vector compressed sensing model. The proposed method constrains the real and \\ imaginary parts of the recovered signal to have the same sparsity profile. It is applied to a compressed \\ sensing through-the-wall radar imaging problem. Experiments based on synthetic data shows that the \\ proposed method achieves lower reconstruction error than the existing CS method.

\section{Keywords} \\ application, signals, valued, imaging, complex, radar, method, sensing, compressed, wall \\ Disciplines \\ Engineering | Science and Technology Studies

\section{Publication Details} \\ F. Tivive \& A. Bouzerdoum, "A compressed sensing method for complex-valued signals with application to \\ through-the-wall radar imaging," in Proceedings of the 38th International Conference on Acoustics, \\ Speech, and Signal Processing, 2013, pp. 2144-2148.
}




\title{
A COMPRESSED SENSING METHOD FOR COMPLEX-VALUED SIGNALS WITH APPLICATION TO THROUGH-THE-WALL RADAR IMAGING
}

\author{
Fok Hing Chi Tivive and Abdesselam Bouzerdoum \\ School of Electrical, Computer and Telecommunications Engineering, \\ University of Wollongong, \\ Northfields Avenue, Wollongong NSW 2522, Australia \\ (e-mail: \{tivive, a.bouzerdoum\}@uow.edu.au)
}

\begin{abstract}
In this paper, we present a compressed sensing method for complexvalued signals based on multiple measurement vector compressed sensing model. The proposed method constrains the real and imaginary parts of the recovered signal to have the same sparsity profile. It is applied to a compressed sensing through-the-wall radar imaging problem. Experiments based on synthetic data shows that the proposed method achieves lower reconstruction error than the existing CS method.
\end{abstract}

Index Terms - Complex-valued, Compressive sensing, Multiple measurement vector

\section{INTRODUCTION}

Over the past few years, compressed sensing (CS) has gained increased interest and has provided a new framework for signal acquisition without the constraint of Nyquist sampling rate. Compressed sensing has shown that a signal or an image, which has a sparse representation in a certain domain, can be reconstructed with far fewer non-adaptive measurements than Nyquist sampling theorem [1]. With the ability of sensing and compressing simultaneously, compressed sensing has been applied to many radar imaging areas, such as Ground Penetrating Radar (GPR) [2,3], Synthetic Aperture Radar (SAR) [4-6], Inverse Synthetic Aperture Radar (ISAR) [7, 8], and Through-the-Wall Radar Imaging (TWRI) [9-12]. Many sparse recovery algorithms have been proposed to solve CS related problems for real-valued signals. Some of the sparse recovery algorithms can cope with complex-valued signals, e.g., the optimization package CVX developed by Grant and Boyd based on convex programming $[13,14]$ and YALL1 package developed by Yang and Zhang [16]. In [15], the authors proposed a general $l_{p}$ minimization recovery algorithm for $\mathrm{CS}$ in which a smoothing constraint is applied on the phase of the signal and a sparse transform is used for the data to improve the signal recovery.

In order to use the existing sparse recovery algorithms for complex-valued CS problem, the real and imaginary parts of the measured input signal are decoupled and concatenated into a column vector. Similarly, the dictionary is also decoupled into real and imaginary sub-matrices to generate a matrix which is four times the size of its original size. The solution obtained from the sparse recovery algorithm comprises the real and imaginary parts, arranged in a similar manner as the measured input signal. This CS strategy has been applied to many CS related problems, such as TWRI $[9,10]$, SAR [17], channel estimation [18], and inverse scattering [19]. However, this strategy has a drawback is that the real and imaginary parts of the recovered signal do not share a common sparsity profile. This issue was mentioned by Ender who applied a standard simplex algorithm to cope with this problem [20].

In this paper, we propose a CS strategy that constrains the real and imaginary parts of the solution to share a common support. The proposed strategy uses the row sparsity constraint of Multiple Measurement Vector (MMV) in CS to force the real and imaging parts of the solution to have the same sparsity profile. We evaluate its effectiveness by applying the proposed CS method to compressed sensing through-the-wall radar imaging, which deals with complexvalued signals.

The remainder of this paper is organized as follows. Section 2 presents an overview of compressed sensing and the existing strategy for converting from complex-valued to real-valued CS model. Section 3 describes the proposed CS method followed by some simulations. Application to TWRI is presented in Section 4. Experimental results are presented in Section 5. Finally, a conclusion is given in Section 6.

\section{COMPRESSED SENSING}

In this section, we briefly describe the existing strategy for solving a complex-valued compressed sensing problem. Consider a complexvalued signal $\boldsymbol{x} \in \mathbb{C}^{N}$ and an orthornormal basis $\Psi \in \mathbb{C}^{N \times N}$, such that $\boldsymbol{x}=\Psi \boldsymbol{\alpha}$, where $\boldsymbol{\alpha} \in \mathbb{C}^{N}$ is a sparse vector. The signal is considered to be sparse if it contains only $K$ nonzero coefficients, where $K \ll N$, or to be compressible if its ordered set of coefficients decays rapidly and the signal can be well approximated by just the first $K$ coefficients.

Compressed sensing shows that a signal that is sparse, or compressible, in some basis can be acquired using a low-rate acquisition process. The signal $\boldsymbol{x}$ is measured through the following model:

$$
\boldsymbol{y}=\Phi \boldsymbol{x}+\boldsymbol{e},
$$

where $\boldsymbol{y} \in \mathbb{C}^{M}$ denotes the measurement vector, $\Phi \in \mathbb{C}^{M \times N}, M<$ $N$, is the sensing matrix, and $e \in \mathbb{C}^{M}$ represents the observation noise. A common approach to recover the original signal $\boldsymbol{x}$ from $\boldsymbol{y}$ is to solve the so-called Basis Pursuit denoising problem given by

$$
\min _{\boldsymbol{\alpha}}\|\boldsymbol{\alpha}\|_{1}, \quad \text { subject to }\|\boldsymbol{y}-\Phi \Psi \boldsymbol{\alpha}\|_{2} \leq \epsilon,
$$

where $\epsilon$ is an upper bound of the $\ell_{2}$-norm of the noise. The CS problem given in (2) can be solved if the sensing matrix satisfies the Restricted Isometry Property (RIP). In the CS literature, there are several types of sparse recovery algorithms that have been proposed to solve this type of minimization problems. Most of them were 
developed for real-valued signals. Therefore, to apply an existing real-valued spare recovery algorithm to a complex-valued signal, the measured signal $\boldsymbol{y}$ and the dictionary $D$ are decoupled into their real and imaginary parts as follows:

$$
\begin{gathered}
\widetilde{D}=\left[\begin{array}{cc}
\Re\{D\} & -\Im\{D\} \\
\Im\{D\} & \Re\{D\}
\end{array}\right] \\
\widetilde{\boldsymbol{y}}=\left[\begin{array}{c}
\Re\{\boldsymbol{y}\} \\
\Im\{\boldsymbol{y}\}
\end{array}\right],
\end{gathered}
$$

and

$$
\widetilde{\boldsymbol{\alpha}}=\left[\begin{array}{l}
\boldsymbol{\alpha}^{r} \\
\boldsymbol{\alpha}^{i}
\end{array}\right],
$$

where $D=\Phi \Psi$ is the dictionary, and $\Re\{\cdot\}$ and $\Im\{\cdot\}$ are the real and imaginary operators, respectively. The vector $\widetilde{\boldsymbol{\alpha}}$ is a concatenation of the real $\boldsymbol{\alpha}^{r}$ and imaginary $\boldsymbol{\alpha}^{i}$ parts of the recovered signal. Here, this decomposition is termed as single measurement vector (SMV) strategy as the CS model contains only one measurement vector. Based on this decomposition, the $\ell_{1}$-norm minimization problem can be rewritten as

$$
\min _{\widetilde{\boldsymbol{\alpha}}}\|\widetilde{\boldsymbol{\alpha}}\|_{1}, \quad \text { subject to } \quad\|\widetilde{\boldsymbol{y}}-\widetilde{\mathbf{D}} \widetilde{\boldsymbol{\alpha}}\|_{2} \leq \epsilon .
$$

The real and imaginary parts of the sparse solution obtained by solving (6) are not guaranteed to have the same sparsity profile. To enforce a constraint on the real and imaginary parts of the sparse solution, we propose to solve (6) as a multiple measurement vector (MMV) problem.

\section{PROPOSED COMPRESSED SENSING METHOD}

Multiple measurement vector (MMV) is a natural extension of single measurement vector (SMV) where a CS problem has several measurement vectors with an over-complete dictionary. The MMV model addresses the recovery of a set of sparse signal vectors that share a common support. In other words, each column of the solution matrix has the same sparsity profile. By using this constraint, we propose to solve (6) as a MMV minimization problem so that the nonzero coefficients in the real and imaginary parts of the recovered complex-valued signal share the same set of indices. Mathematically, the proposed CS method can be described as follows. First, the measurement vector $\boldsymbol{y}$ is duplicated. Both measurement vectors including the replicated one are decoupled and rearranged as columns of the $Y$ matrix:

$$
Y=\left[\begin{array}{cc}
\Re\{\boldsymbol{y}\} & -\Im\{\boldsymbol{y}\} \\
\Im\{\boldsymbol{y}\} & \Re\{\boldsymbol{y}\}
\end{array}\right] .
$$

The sparse matrix solution can be written as

$$
\Omega=\left[\begin{array}{ll}
\phi_{1}^{r} & \phi_{2}^{i} \\
\phi_{1}^{i} & \phi_{2}^{r}
\end{array}\right],
$$

where $\phi_{1}^{r}$ and $\phi_{1}^{i}$ are, respectively, the real and imaginary parts of the solution for the first column of $Y$. Similarly, $\phi_{2}^{r}$ and $\phi_{2}^{i}$ are the real and imaginary parts of the solution for the second column of $Y$. This decomposition is termed as MMV strategy. Now, we can write

$$
\min _{\Omega}\|\Omega\|_{1}, \quad \text { subject to } \quad\|Y-\widetilde{D} \Omega\|_{2} \leq \epsilon,
$$

Then, a sparse recovery algorithm designed for MMV, such as, simultaneous Orthogonal Matching Pursuit (S-OMP) [21, 22] or MFOCUSS [23] can be used for signal recovery. The real and imaginary parts of the recovered signal $\boldsymbol{\alpha}^{\star}$ can be calculated as

$$
\begin{aligned}
& \Re\left\{\boldsymbol{\alpha}^{\star}\right\}=\left(\phi_{1}^{r}+\phi_{2}^{r}\right) / 2, \\
& \Im\left\{\boldsymbol{\alpha}^{\star}\right\}=\left(\phi_{1}^{i}-\phi_{2}^{i}\right) / 2 .
\end{aligned}
$$

To test its effectiveness, the proposed method is applied to the recovery of a complex-valued signal. The complex-valued signal is generated to have a length of $N=500$ where $16 \%$ of the coefficients are nonzero. For the recovery of the signal, a dictionary of size $200 \times 500$ is designed with its complex-valued elements are independent and identically distributed variables drawn from a Gaussian distribution $\mathcal{N}(0,1)$. The measurement vector $\boldsymbol{y}$ is defined as $\boldsymbol{y}=D \boldsymbol{\alpha}$ and is corrupted by white Gaussian noise. OMP is used as a sparse recovery algorithm in conjunction with the SMV strategy and the proposed MMV strategy. The recovery performance of the algorithm is measured in terms of the normalized mean square error (NMSE) given by

$$
\mathrm{NMSE}=\frac{\left\|\boldsymbol{\alpha}-\boldsymbol{\alpha}^{\star}\right\|_{2}}{\|\boldsymbol{\alpha}\|_{2}}
$$

The simulation was performed over 1000 runs and the indices of the nonzero coefficients of the recovered signal were randomly chosen for each run. The averaged normalized mean square error as a function of the number of measurements and the SNR of the input signal are shown in Figs. 1(a) and (b). Figure 1(a) shows the proposed MMV strategy gives lower reconstruction error than the SMV strategy when increasing the number of measurements. Using noisy measurements, we show that the proposed MMV strategy is more robust against noise than the SMV strategy, cf. Fig. 1(b). In the following section, we apply the proposed CS method to compressed sensing through-the-wall radar imaging.
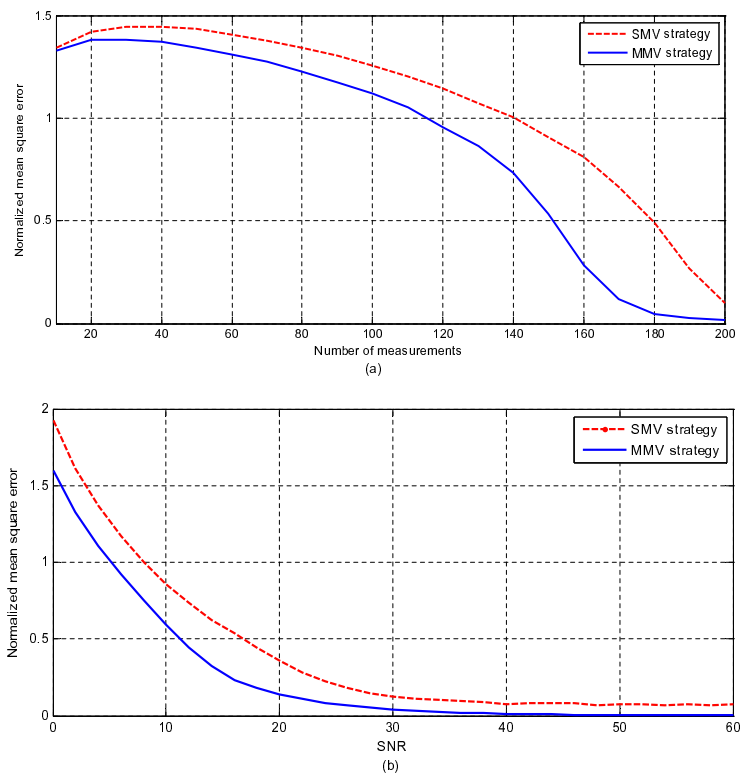

Fig. 1. Reconstruction error of the SMV and the proposed MMV strategies: (a) NMSE as a function of the number of measurements and (b) NMSE as a function of the SNR of the measured signal. 


\section{THROUGH-THE-WALL RADAR IMAGING}

Through-the-wall radar imaging (TWRI) has been given much attention recently due its wide range of applications in public safety, law enforcement and military [24]. In order to achieve high resolution imaging, large signal bandwidth and array aperture are required, leading to an increase in data acquisition time. There is an increasing interest in fast data acquisition and image formation for TWRI to allow prompt actionable intelligence and to enable reliable situational awareness. To achieve this objective, compressed sensing TWRI methods $[9,10]$ were developed. In these methods, a real-valued sparse recovery algorithm in combination with the SMV strategy was used for image reconstruction. In the following, the signal model and compressed sensing TWRI are described.

\subsection{Signal Model}

In a stepped-frequency TWRI approach, a large bandwidth is achieved by transmitting $M_{f}$ narrowband signals of frequency,

$$
f_{m}=f_{m}+m \Delta f, \quad \text { for } m=0, \ldots, M_{f}-1,
$$

where $f_{0}$ is the initial frequency and $\Delta f$ is the frequency step size. Assuming that a $M_{a}$-element array of transceivers is used to interrogate the scene behind the wall. The scene is divided into a rectangular grid of $N_{x}$ by $N_{y}$ pixels. Given $P$ targets in the scene, the stepped-frequency signal of the $m$-th frequency received at the $n$-th antenna $z(m, n)$ is given by

$$
z(m, n)=\sum_{p=1}^{P} \sigma_{p} \exp \left(-j 2 \pi f_{m} \tau_{n, p}\right),
$$

where $n \in\left[0, M_{a}-1\right], m \in\left[0, M_{f}-1\right], \sigma_{p}$ is the reflection coefficient of the $p$-th target, $\tau_{n, p}$ is the two-way propagation delay of the signal from the $n$-th antenna to the $p$-th target. It is assumed that we know the wall parameters, i.e., the wall thickness and dielectric constant, and the received radar signal is free of the wall EM signature by using a wall-clutter mitigation. After the acquisition of all the data samples, delay-and-sum (DS) beamforming, a common image formation method, is used to reconstruct the scene. Let $N=N_{x} N_{y}$ denote the total number of pixels in the formed image. The complex-valued of the $q$-th pixel can be computed as

$$
I(q)=\frac{1}{M_{f} M_{a}} \sum_{m=0}^{M_{f}-1} \sum_{n=0}^{M_{a}-1} y(m, n) \exp \left(j 2 \pi f_{m} \tau_{n, q}\right),
$$

where $q \in[1, N], \tau_{n, q}$ denotes the focusing delay applied to the output of the $n$-th transceiver for the $q$-th pixel. The computation of the focusing delay for TWRI can be found in [25]. With DS beamforming, all the data measurements are required to form highresolution radar images, at the expense of increasing the data acquisition time and data storage requirements.

\subsection{Compressed Sensing Through-the-Wall Radar Imaging}

Compressed sensing has been applied to reconstruct a radar image accurately with few measurements. Since the number of targets is far fewer than the number of pixels, the target space can be considered as sparse. Thus, the image formation problem in TWRI can be formulated as a CS problem. Let $s_{i}$ denote an indicator function, defined as

$$
s_{q}= \begin{cases}\sigma_{p}, & \text { if a target } p \text { exists at the } q \text {-th pixel location; } \\ 0, & \text { otherwise. }\end{cases}
$$

Using the indicator function, we define a column vector $s=$ $\left[s_{1}, \ldots, s_{q}\right]$, where $q \in[1, N]$. We also arrange the measurements given in (14) into a vector $\boldsymbol{z} \in \mathbb{C}^{M}, M=M_{f} M_{a}$, by concatenating all $M$ measurements into a column vector:

$$
\boldsymbol{z}=\left[z(0,0), \ldots, z\left(M_{f}-1, M_{a}-1\right)\right]^{T},
$$

Therefore, Equation (14) can be rewritten as

$$
z=\Psi s
$$

where $\Psi \in \mathbb{C}^{M \times N}$. Each element of the matrix $\Psi$ is given by

$$
\Psi(i, q)=\exp \left(-j 2 \pi f_{m} \tau_{n, q}\right),
$$

with $m=i \bmod M_{f}, n=\left\lfloor i / M_{f}\right\rfloor$ and $i=0, \ldots, M_{f} M_{a}-1$. Suppose that we select a few measurements using a measurement matrix, $\Phi \in \mathbb{R}^{J \times M N},(J<M \ll N)$, where $J$ is the total number of selected measurements. Then, the measured data $\boldsymbol{y}$ can be expressed as

$$
\boldsymbol{y}=\Phi \boldsymbol{z}=D \boldsymbol{s}
$$

where $D=\Phi \Psi$. It follows that (20) is an underdetermined system of linear equations that has infinitely many solutions. However, it is possible to recover the sparse signal $s$ through compressive sensing when the matrix $D$ satisfies the Restricted Isometry Property (RIP) [1]:

$$
\left(1-\delta_{K}\right)\|\boldsymbol{s}\|_{2}^{2} \leq\|D \boldsymbol{s}\|_{2}^{2} \leq\left(1+\delta_{K}\right)\|\boldsymbol{s}\|_{2}^{2},
$$

where the sparse vector $s$ has $K$ nonzero coefficients and $\delta_{K} \in$ $(0,1)$. A small value of $\delta_{K}$ leads to better construction of the sparse signal. It has been shown that to recover a $K$-sparse signal $s$ exactly or with high probability, the number of measurements $J$ has to satisfy

$$
J \geq c K \log (W / K),
$$

where $c$ is small constant and $W$ is the dimension of the vector $s$ [6]. With the measured data $\boldsymbol{y}$ and the dictionary $D$, the signal $s$ can be recovered from the solution of a convex optimization problem based on $\ell_{1}$-norm:

$$
\min \|\boldsymbol{s}\|_{1} \text { subject to }\|\boldsymbol{y}-D \boldsymbol{s}\|_{2} \leq \varepsilon,
$$

where $\|\boldsymbol{s}\|_{1}=\sum_{i}\left|s_{i}\right|$ and $\varepsilon$ bounds the amount of noise in the measured data.

\section{EXPERIMENTAL RESULTS}

The proposed CS method is compared with the existing CS method on synthetic TWR data. For simulations, the TWRI scene comprises three targets that are located at $[0,1],[-1,2.5]$, and $[1.5,3] \mathrm{m}$ behind a homogeneous wall. Each target is represented by a group of four by four pixels. The wall has a thickness of $0.15 \mathrm{~m}$ and a dielectric constant of 7.6. A two meter line array containing 61 antenna elements is placed at a standoff distance of $1 \mathrm{~m}$ from the wall. Each antenna transmits and receives a stepped-frequency signal with a bandwidth of $2 \mathrm{GHz}$ centered at $2.5 \mathrm{GHz}$. The frequency step size is set to $5 \mathrm{MHz}$, producing 401 frequency samples. We assume that the wall backscatters have been removed from the radar returns using a wall-clutter mitigation method, such as background subtraction, spatial filtering [26], or the SVD-based wall clutter mitigation method [27, 28]. Illuminating the scene with all the antennas and frequencies, we have a set of 24,461 measurements, i.e., 401 frequencies times 61 antenna locations. Based on compressive sensing, a subset of these measurements is required to accurately recovery the target space. Let us consider the target space be a square grid 
with 50 rows and 50 columns, i.e., an image of size $50 \times 50$ pixels. We use the target-to-clutter ratio (TCR) to measure the quality of the formed image. TCR is computed as

$$
\mathrm{TCR}=20 \log \left(\frac{\frac{1}{N_{t}} \sum_{q \in R_{t}}|I(q)|}{\frac{1}{N_{c}} \sum_{q \in R_{c}}|I(q)|}\right),
$$

where $R_{t}$ is the target region, $R_{c}$ is the clutter region, $N_{c}$ and $N_{t}$ are, respectively, the number of pixels in the clutter and target regions. To reconstruct the scene, we use $5 \%$ of the total data volume, where the frequencies are randomly chosen and the antenna locations are chosen equispaced across the array. Orthogonal matching pursuit is used as a sparse recovery algorithm since it is a fast technique to obtain a sparse solution. The stopping criterion for OMP was set to 100 iterations. In other words, OMP only computes the first 100 nonzero coefficients of the solution. Figure 2(a) shows the image reconstructed by DS beamforming based on the full data volume for a noise-free scene with three targets behind the wall. Figures 2(b) and (c) illustrate the images formed by the CS imaging method in combination with the SMV strategy and the proposed MMV strategy, respectively. It is clear that the existing SMV strategy produces an image with many false target pixels. This is due to the fact that the real and imaginary parts of the solution obtained from OMP do not have the same sparsity profile. On the other hand, the proposed strategy, which employs the row sparsity constraint of MMV CS model, generates a radar image with few false target pixels. In terms of image quality, DS beamforming achieves a TCR of $19.94 \mathrm{~dB}$ and the CS imaging method with the SMV strategy gives a TCR of $25.43 \mathrm{~dB}$. Using the proposed MMV strategy, we obtain a TCR of $29.56 \mathrm{~dB}$.

\section{CONCLUSION}

In this paper, a method for complex-valued CS problem was proposed. The proposed CS method constrains the real and imaginary parts of the solution to share a common support by using the row sparsity condition of MMV CS model. For compressed sensing TWRI, the proposed CS method, based on simulated data, produced a radar image with fewer false target pixels than the existing CS method using the same sparse recovery algorithm.

\section{Acknowledgment}

This work is supported in part by a grant from the Australian Research Council (ARC).

\section{REFERENCES}

[1] E. J. Candès and M. B. Wakin, "An introduction to compressive sampling: A sensing/sampling paradigm that goes against the common knowledge in data acquisition," IEEE Signal Processing Magazine, vol. 25, no. 2, pp. 21-30, 2008.

[2] A. C. Gurbuz, J. H. McClellan, and W. R. Scott, "Compressive sensing for subsurface imaging using ground penetrating radar," Signal Processing, vol. 89, no. 10, pp. 1959-1972, 2009.

[3] A. C. Gurbuz, J. H. McClellan, and W. R. Scott, "A compressive sensing data acquisition and imaging method for stepped frequency GPRS," IEEE Transactions on Signal Processing, vol. 57, no. 7, pp. 2640-2650, 2009.

[4] M. A. Herman and T. Strohmer, "High-resolution radar via compressed sensing," IEEE Transactions on Signal Processing, vol. 57, no. 6, pp. 2275-2284, 2009.

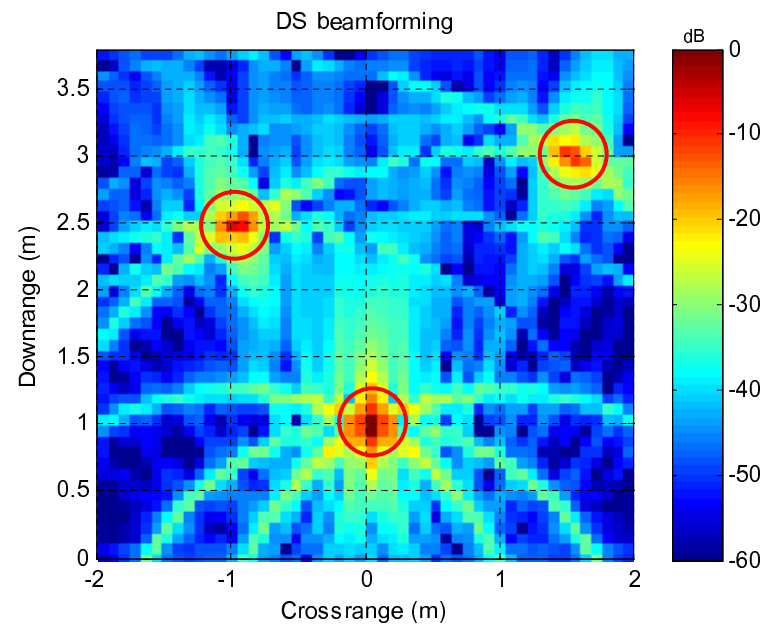

(a)

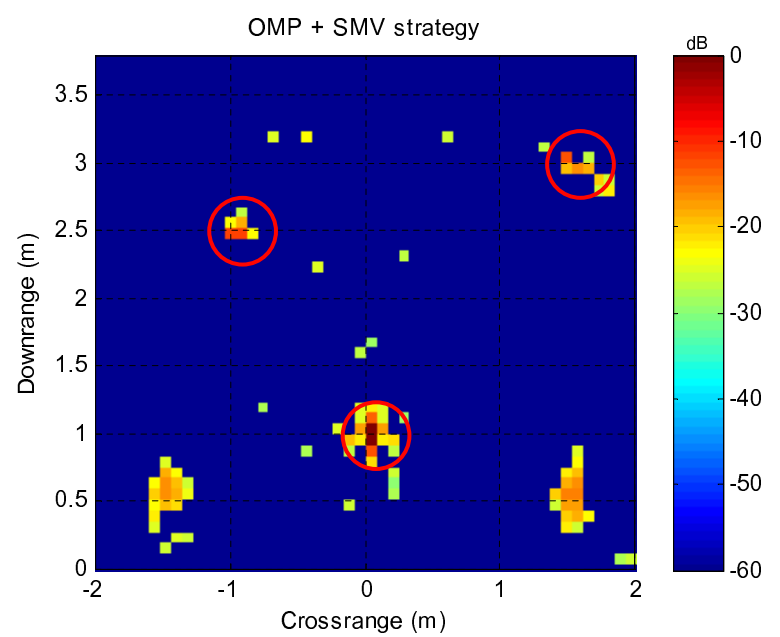

(b)

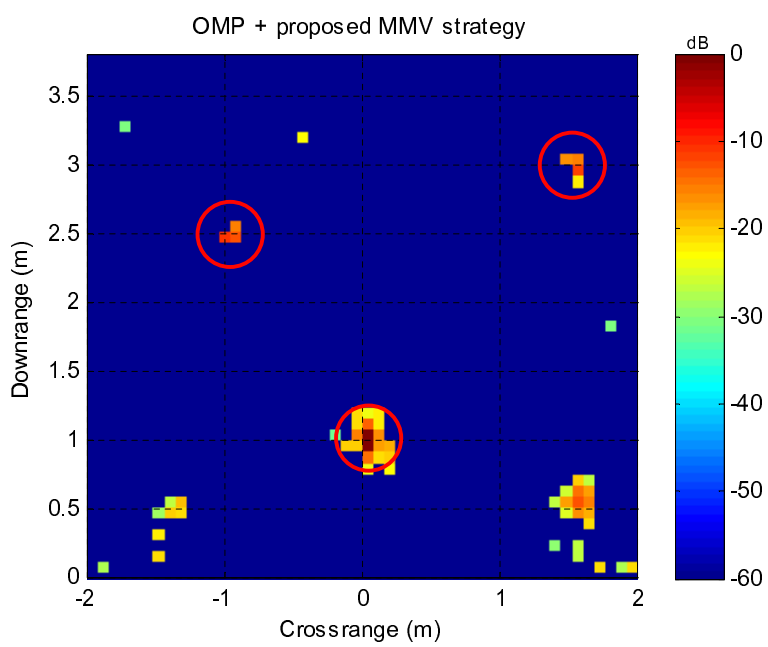

(c)

Fig. 2. Image formed by an imaging method: (a) DS beamforming, (b) OMP + SMV strategy and (b) OMP + proposed MMV strategy. 
[5] L.C. Potter, E. Ertin, J. T. Parker, and M. Cetin, "Sparsity and compressed sensing in radar imaging," Proceedings of the IEEE, vol. 98 , no. 6, 2010.

[6] R. Baraniuk and P. Steeghs, "Compressive radar imaging," in IEEE Radar Conference, 2007, pp. 128-133.

[7] L. Zhang, Z.-J. Qiao, M. Xing, Y. Li, and Z. Bao, "Highresolution ISAR imaging with sparse stepped-frequency waveforms," IEEE Transactions on Geoscience and Remote Sensing, vol. 49, no. 11, pp. 4630-4651, 2011.

[8] H. Wang, Y. Quan, M. Xing, and S. Zhang, "ISAR imaging via sparse probing frequencies," IEEE Geoscience and Remote Sensing Letters, vol. 8, no. 3, pp. 451-455, 2011.

[9] Y.-S. Yoon and M. G. Amin, "Compressed sensing technique for high-resolution radar imaging," in Proc. of the SPIE, Signal Processing, Sensor Fusion, and Target Recognition XVII, vol. 6968, 2008, pp. 69681A-69681A-10.

[10] Q. Huang, L. Qu, B. Wu, and G. Fang, "UWB through-wall imaging based on compressive sensing," IEEE Transactions on Geoscience and Remote Sensing, vol. 48, no. 3, pp. 1408-1415, 2010.

[11] W. Zhang, M. G. Amin, F. Ahmad, A. Hoorfar, and G. E. Smith, "Ultrawideband impulse radar through-the-wall imaging with compressive sensing," International Journal of Antennas and Propagation, vol. 2012, no. Article ID 251497, pp. 1-11, 2012.

[12] M. Leigsnering, C. Debes, and A. M. Zoubir, "Compressive sensing in through-the-wall radar imaging," in IEEE International Conference on Acoustics, Speech and Signal Processing, 2011, pp. 4008- 4011.

[13] M. Grant and S. Boyd, Graph implementations for nonsmooth convex programs, Recent Advances in Learning and Control (a tribute to M. Vidyasagar), V. Blondel, S. Boyd, and H. Kimura, editors, pp. 95-110, Lecture Notes in Control and Information Sciences, Springer, 2008.

[14] CVX Research, Inc. CVX: Matlab software for disciplined convex programming, version 2.0 beta. http://cvxr.com/cvx, September 2012.

[15] S. Yu, S. Khwaja, and J. Ma, "Compressed sensing of complexvalued data," in Signal Processing, vo. 92, no. 2, pp. 357-362, 2012.

[16] J. Yang and Y. Zhang, "Alternating direction algorithms for 11problems in compressive sensing," SIAM Journal on Scientific Computing, vol. 33, no. 1-2, pp. 250-278, 2011.

[17] I. Stojanovic, W. C. Karl, and M. Cetin, "Compressed sensing of mono-static and multi-static sar," in Proc. SPIE 7337, Algorithms for Synthetic Aperture Radar Imagery XVI, 733705 (April 28, 2009); doi:10.1117/12.818808.

[18] G. Taubock and F. Hlawatsch, "A compressed sensing technique for ofdm channel estimation in mobile environments: Exploiting channel sparsity for reducing pilots," in IEEE International Conference on Acoustics, Speech and Signal Processing, 2008, pp. 2885-2888.

[19] E. A. Marengo, "Inverse scattering by compressive sensing and signal subspace methods," in IEEE International Workshop on Computational Advances in Multi-Sensor Adaptive Processing, 2007, pp. 109-112.
[20] J. H. G. Ender, "On compressive sensing applied to radar," Journal Signal Processing, vol. 90, no. 5, pp. 1402-1414, 2010.

[21] J. Chen and X. Huo, "Theoretical results on sparse representations of multiple measurement vectors," IEEE Transactions on Signal Processing, vol. 54, no. 12, pp. 4634-4643, 2006.

[22] J. Tropp, A. Gilbert, and M. Strauss, "Algorithms for simultaneous sparse approximation. part I: Greedy pursuit," Signal Processing, vol. 86, no. 3, pp. 572-588, 2006.

[23] S. Cotter, B. Rao, K. Engan, and K. Kreutz-Delgado, "Sparse solutions to linear inverse problems with multiple measurement vectors," IEEE Transactions on Signal Processing, vol. 53, no. 7, pp. 2477-2488, 2005.

[24] M. G. Amin, (Ed.), Through-the-wall radar imaging, CRC Press, Boca Raton, FL, 2011.

[25] F. Ahmad, M. G. Amin, and S. A. Kassam, "Synthetic aperture beamformer for imaging through a dielectric wall," IEEE Transactions on Aerospace and Electronic Systems, , no. 1, pp. 271-283, 2005.

[26] Y.-S. Yoon and M. G. Amin, "Spatial filtering for wall-clutter mitigation in through-the-wall radar imaging," IEEE Transactions on Geoscience and Remote Sensing, vol. 47, no. 9, pp. 3192-3208, 2009.

[27] F. H. C. Tivive, M. G. Amin, and A. Bouzerdoum, "Wall clutter mitigation based on eigen-analysis in through-the-wall radar imaging," in Proc. of International Conference on Digital Signal Processing, 2011, pp. 1-8.

[28] F. H. C. Tivive, A. Bouzerdoum, and M. G. Amin, "An SVDbased approach for mitigating wall reflections in through-thewall radar imaging," in Proc. IEEE Radar Conference, 2011, pp. 519-524. 